\title{
Enhancement of cellular uptake, transport and oral absorption of protease inhibitor saquinavir by nanocrystal formulation
}

\author{
Yuan $\mathrm{HE}^{1}$, Deng-ning $\mathrm{XIA}^{2}$, Qiu-xia $\mathrm{LI}^{1,2}$, Jin-song $\mathrm{TAO}^{2,3}$, Yong $\mathrm{GAN}^{2, *}$, Chi WANG ${ }^{1, *}$ \\ ${ }^{1}$ School of Pharmacy, Chongqing Medical University, Chongqing 400016, China; ${ }^{2}$ Shanghai Institute of Materia Medica, Chinese \\ Academy of Sciences, Shanghai 201203, China; ${ }^{3}$ Department of Pharmacy, College of Clinical Medicine, Nanchang University, \\ Nanchang 330047, China
}

\begin{abstract}
Aim: Saquinavir (SQV) is the first protease inhibitor for the treatment of HIV infection, but with poor solubility. The aim of this study was to prepare a colloidal nanocrystal suspension for improving the oral absorption of SQV.

Methods: SQV nanocrystals were prepared using anti-solvent precipitation-high pressure homogenization method. The nanocrystals were characterized by a Zetasizer and transmission electron microscopy (TEM). Their dissolution, cellular uptake and transport across the human colorectal adenocarcinoma cell line (Caco-2) monolayer were investigated. Bioimaging of ex vivo intestinal sections of rats was conducted with confocal laser scanning microscopy. Pharmacokinetic analysis was performed in rats administered nanocrystal SQV suspension (50 mg/kg, ig), and the plasma SQV concentrations were measured with HPLC.

Results: The SQV nanocrystals were approximately $200 \mathrm{~nm}$ in diameter, with a uniform size distribution. The nanocrystals had a rod-like shape under TEM. The dissolution, cellular uptake, and transport across a Caco-2 monolayer of the nanocrystal formulation were significantly improved compared to those of the coarse crystals. The ex vivo intestinal section study revealed that the fluorescently labeled nanocrystals were located in the lamina propria and the epithelium of the duodenum and jejunum. Pharmacokinetic study showed that the maximal plasma concentration $\left(C_{\max }\right)$ was 2.16-fold of that for coarse crystalline SQV suspension, whereas the area under the curve (AUC) of nanocrystal SQV suspension was 1.95-fold of that for coarse crystalline SQV suspension.

Conclusion: The nanocrystal drug delivery system significantly improves the oral absorption of saquinavir.
\end{abstract}

Keywords: saquinavir; protease inhibitor; nanocrystals; Caco-2 cell monolayer; intestinal mucosa; pharmacokinetics

Acta Pharmacologica Sinica (2015) 36: 1151-1160; doi: 10.1038/aps.2015.53; published online 10 Aug 2015

\section{Introduction}

Poor aqueous solubility is a major hurdle in achieving adequate bioavailability for a majority of new drug candidates and for drugs in clinical use. It has been reported that approximately $40 \%$ of marketed drugs have low solubility, while approximately $80 \%-90 \%$ of drug candidates in development could fail because of solubility problems ${ }^{[1]}$. Thus, the development of safe and effective formulations that improve the oral absorption of poorly soluble drugs may increase the success rate of drugs in development.

Colloidal nanodrug formulations (nanocrystal suspensions) are typically composed of drug particles with an average diameter of less than $1 \mu \mathrm{m}$ and stabilizers (surfactants

\footnotetext{
* To whom correspondence should be addressed.

E-mail simm2122@vip.sina.com (Yong GAN); Wchi639@sohu.com (Chi WANG)

Received 2015-03-10 Accepted 2015-04-23
}

or polymers). The dissolution rate of drugs is proportional to the surface area available for dissolution, as described by the Noyes-Whitney equation. According to the OstwaldFreundlich equation, an increase in the surface area can also be expected to increase the equilibrium solubility of a drug ${ }^{[2]}$. Thus, a nanocrystal formulation is generally considered an effective strategy for enhancing the solubility of poorly soluble or insoluble drugs ${ }^{[3,4]}$. Nanocrystals possess a drug loading capacity of almost $100 \%$ in contrast to matrix nanoparticles such as polymeric nanoparticles ${ }^{[5]}$, liposomes ${ }^{[6]}$, and solid lipid nanoparticles ${ }^{[7]}$. The high drug loading capacity and enhanced drug dissolution make nanocrystals an efficient drug delivery system into or across cells, thereby reaching a therapeutic plasma concentration.

However, our understanding of the oral absorption of nanocrystals has not greatly improved over the past two decades. In particular, there is a lack of microscopic evidence to show that nanocrystals are internalized in their intact form or are 
transported as dissolved molecules with subsequent accumulation within the cells. Intestinal absorption mechanisms have been proposed to be related to the enhanced dissolution rate and improved bioadhesion of nanocrystals due to their large surface area ${ }^{[8]}$ and absorption through mesenteric lymph transport ${ }^{[9]}$. However, the fate of nanosuspensions within the body is still poorly understood.

Saquinavir (SQV) is the first protease inhibitor to be approved for the treatment of HIV infection. Over the last decade, SQV has become a major component of combination HIV chemotherapies, which are highly active antiretroviral therapies ${ }^{[10,11]}$. The molecular weight of SQV is 670.8 , and its partition coefficient $(\log P)$ is 4.1 . The solubility of SQV is highly $\mathrm{pH}$-dependent, and the drug is usually administered in its salt form (SQV mesylate) ${ }^{[12]}$. The solubility of SQV mesylate at $\mathrm{pH} 3.5$ is $2.27-2.70 \mathrm{mmol} / \mathrm{L}$. This solubility is much higher than that of the base $(0.07-1.98 \mathrm{mmol} / \mathrm{L})$ over the same $\mathrm{pH}$ range ${ }^{[12]}$. Although the free base exhibits superior solubility at a low $\mathrm{pH}$, the solubility decreases as the $\mathrm{pH}$ increases. Solubility would be especially poor in the intestine, where the physiological $\mathrm{pH}$ is above 5. Thus, low solubility is still a limiting factor in the oral absorption of SQV. In addition to its poor solubility, SQV is metabolized by both human hepatic and small intestinal enzymes, which results in low oral bioavailability $(0.7 \%-4.0 \%)$, depending upon the dosage form ${ }^{[13,14]}$.

The aim of the present study was to prepare a colloidal nanodrug suspension to enhance the oral absorption of SQV. A stable suspension of nanocrystals was prepared by a precipitation-high pressure homogenization method. The physical properties of the prepared SQV nanocrystals were characterized by transmission electron microscopy (TEM) and X-ray powder diffraction (XRPD). The dissolution rate of the nanocrystals was also determined. The absorption of the nanocrystals were studied in Caco-2 cells and an ex vivo rat intestinal tract model. In addition, the oral bioavailability of the nanosuspension was evaluated in Sprague-Dawley rats. Therefore, in the present study, we focused on the development of nanocrystals as an oral formulation aimed at improving the oral bioavailability of SQV.

\section{Materials and methods Materials}

Saquinavir (SQV) mesylate was obtained from Chembest Research Laboratories (Shanghai, China). Poly (sodium 4-styrenesulfonate) (PSS) was purchased from Sigma-Aldrich (St Louis, MO, USA). Ethyl rhodamine B was obtained from Sinopharm Chemical Reagent Co, Ltd (Shanghai, China). All other chemicals and reagents were commercially obtained and were of analytical grade.

\section{Cell culture}

Caco-2 cells were cultured and passaged in Dulbecco's modified Eagle's medium (DMEM; Gibco-Life; Grand Island, NY, USA) supplemented with $4500 \mathrm{mg} / \mathrm{L}$ high glucose, $10 \%$ fetal bovine serum, 100 units $/ \mathrm{mL}$ penicillin, and $100 \mu \mathrm{g} / \mathrm{mL}$ streptomycin at $37^{\circ} \mathrm{C}$ and $5 \% \mathrm{CO}_{2}$.

\section{Animal preparation}

Male Sprague-Dawley rats (body weight, 250-280 g) were provided by SLAC Lab Animal Ltd (Shanghai, China). The experiments were performed under guidelines approved by the Institutional Animal Care and Use Committee of the Shanghai Institute of Materia Medica, Chinese Academy of Sciences (IACUC, 2013-07-GY-12). The rats were maintained on a $12 \mathrm{~h}$ light/dark cycle and given fresh rat chow daily with free access to food and water. The rats were acclimated for at least $5 \mathrm{~d}$ prior to the experiments. The rats were fasted for $12 \mathrm{~h}$ prior to the experiments with free access to water.

\section{Preparation of saquinavir nanocrystals}

SQV nanocrystals were prepared using an anti-solvent precipitation-high pressure homogenization method. Briefly, $400 \mathrm{mg}$ of coarse SQV crystals were dissolved in $2 \mathrm{~mL}$ dimethyl sulfoxide (DMSO). This solution was rapidly added to $50 \mathrm{~mL}$ of phosphate buffered saline (PBS), pH 7.4, containing $0.12 \%$ PSS, using magnetic stirring (500 rounds per minute) to obtain a starting suspension. After anti-solvent precipitation, the suspension was placed in a high pressure homogenizer (ATS Engineering Inc, Shanghai, China) equipped with a cooling device that was connected to a circulating water bath (Julabo F12, Seelbach, Germany) and maintained at $4{ }^{\circ} \mathrm{C}$. The homogenization occurred at 800 bars for 20 cycles, yielding a milky suspension of SQV nanocrystals as shown in Figure 1. The nanocrystal suspension was concentrated by centrifugation at $21752 \times \mathrm{g}$ for $30 \mathrm{~min}$ (Beckman Coulter Allegra 64R centrifuge, USA). The supernatant was removed, and the precipitate was dispersed in $50 \mathrm{~mL}$ of PBS (pH 7.4) containing $0.12 \%$ PSS.

To label the nanocrystals with fluorescein, hybrid nanocrystals containing ethyl rhodamine B incorporated in crystal lattice were prepared according to the method described by Zhao et al (2011), with a slight modification ${ }^{[15]}$. Briefly, $20 \mathrm{mg}$ of ethyl rhodamine and $400 \mathrm{mg}$ of coarse SQV crystals were dissolved in $2 \mathrm{~mL}$ DMSO, and the same process was used as described for the preparation of the SQV nanosuspension.

\section{Characterization of nanocrystals}

The particle size and zeta potential of the SQV nanocrystals were determined using a Zetasizer (Nano-ZS, Malvern instruments, UK). Particle size measurements were performed at $25^{\circ} \mathrm{C}$ at a scattering angle of $173^{\circ}$. The SQV nanosuspensions were diluted five-fold using purified water (Milli-Q Integral, Millipore, USA) prior to determination, and each sample was measured in triplicate. The morphology of the coarse crystals and nanocrystals were observed using a scanning electron microscope (SEM, Agilent 8500, Santa Clara, CA, USA) and TEM (Hitachi H-600, Tokyo, Japan), respectively. After diluting the samples 10-fold with purified water, a drop of the suspension was spread on a copper grid, and the excess liquid was removed with filter paper. The samples were dried at room temperature for $10 \mathrm{~min}$ and observed using TEM.

The crystalline form of the SQV nanocrystals was examined by XRPD using a Bruker AXS D8 Advance diffractometer 

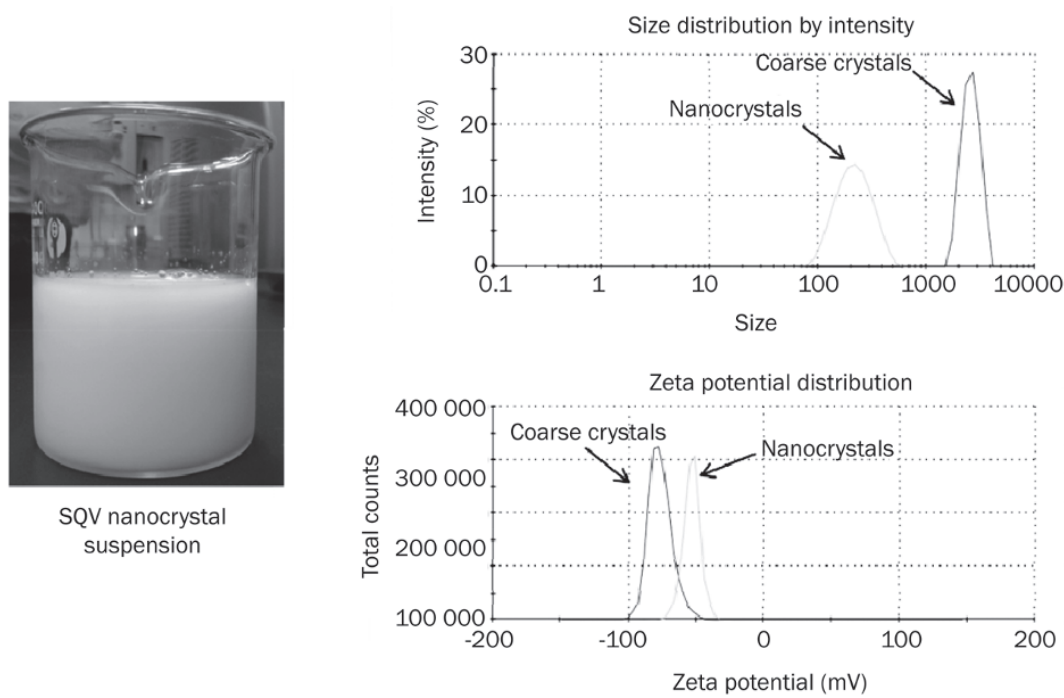

Figure 1. Particle size and zeta-potential of saquinavir (SQV) nanocrystals and coarse crystals.

(Karlsruhe, Germany). The samples were freeze dried before analysis. The samples were placed on zero-background silicon plates and measured at ambient conditions in reflection mode. A continuous $2 \theta$ scan was performed in the range of $5^{\circ}-45^{\circ}$ with a step size of $0.02^{\circ}$ using copper $\mathrm{K}$-a radiation $(\lambda=1.540629 \AA)$ with a scanning speed of $0.067^{\circ} 2 \theta / \mathrm{s}$. The voltage and current applied were $40 \mathrm{kV}$ and $40 \mathrm{~mA}$, respectively. The data were collected and analyzed using DIFFRAC SUITE $^{\text {TM }}$ Software (Bruker).

\section{Drug dissolution study}

Dissolution experiments were performed using the USP35NF30 paddle method (Symphony 7100, Distek, Inc, USA). PBS ( $\mathrm{pH}$ 6.8) was used as the dissolution medium, the temperature was maintained at $37.0 \pm 0.5^{\circ} \mathrm{C}$, and the paddle speed was 100 rounds per minute. Samples containing $5 \mathrm{mg}$ of drug were dispersed in $200 \mathrm{~mL}$ of dissolution medium, and then samples of the solution ( $3 \mathrm{~mL}$ each) were withdrawn at different time and passed through a $0.1 \mu \mathrm{m}$ syringe filter (Millipore Filters, USA). The SQV concentration was quantified using a UV spectrophotometer (Mode TU-1901, Purkinje General, China) at $239 \mathrm{~nm}$. In addition, the fluorescence intensity of ethyl rhodamine B released from the ethyl rhodamine B-stained SQV crystals was determined using a microplate reader (SYNERGY ${ }^{\mathrm{TM}} \mathrm{H} 1$, BioTek, USA) at the excitation and emission wavelengths of $535 \mathrm{~nm}$ and $580 \mathrm{~nm}$, respectively.

\section{Cellular uptake of nanocrystals in Caco-2 cells}

To track the cellular uptake of the nanocrystals, the fluorescein-labeled nanocrystals were prepared by physically incorporating ethyl rhodamine B into the crystal lattice of SQV. This was accomplished using anti-solvent precipitation as mentioned in the previous section. This method (without high-pressure homogenization) was also used to prepare fluorescein-labeled microcrystals with the same particle size as that of the coarse powder. Before the cellular uptake study, the fluorescein intensities of both formulations were adjusted to the same level.

Caco- 2 cells were seeded in 12 -well plates $\left(1 \times 10^{5}\right.$ cells per well) and grown for $5 \mathrm{~d}$, with feeding every $2 \mathrm{~d}$. The fluorescent-labeled nanocrystals were dispersed in Hank's balanced salt solution (HBSS) to form a test suspension (50 $\mu \mathrm{g} / \mathrm{mL}$ ). The cells were pre-incubated with HBSS alone at $37^{\circ} \mathrm{C}$ for $30 \mathrm{~min}$. After removing the HBSS, the cells were exposed to the test suspension $(1 \mathrm{~mL})$ at $37^{\circ} \mathrm{C}$ for $2 \mathrm{~h}$. Then, the test suspension was removed, and the cell layer was washed three times with $2 \mathrm{~mL}$ of HBSS. The cells were fixed with $4 \%$ buffered paraformaldehyde for $10 \mathrm{~min}$, stained with 4',6-diamidino-2-phenylindole (DAPI) (5 $\mathrm{g} / \mathrm{mL}$, Beyotime, Haimen, China), and finally imaged under a confocal laser scanning microscope (CLSM) (FluoView FV10i, Olympus, Japan). The average fluorescence intensity of cells was quantified by ImageJ 1.49n (Wayne Rasband, National Institutes of Health, USA). The fluorescent images were converted to 8-bit before being quantified by threshold analysis ${ }^{[16]}$.

\section{Transport of saquinavir across the Caco- 2 cell monolayers}

Caco- 2 cells were seeded onto Transwell polycarbonate cell culture inserts (12-mm diameter, 3- $\mu \mathrm{m}$ pore size, Costar ${ }^{\circledR}$, Corning Costar Co, Cambridge, MA, USA) at a density of approximately $1 \times 10^{5}$ cells per well. The cells were cultured for 21-25 d to attain confluence and full maturation, including P-glycoprotein (P-gp) expression and the formation of tight junctions in the cell monolayer. The culture medium was replaced every other day for the first week and daily thereafter. The apical (AP) and basolateral (BL) compartments contained 0.5 and $1.5 \mathrm{~mL}$ of culture medium, respectively.

The integrity of the Caco-2 cell monolayers was evaluated both before and immediately after the transport studies using Millicell ${ }^{\circledR}$-ERS (Millipore, Bedford, MA, USA). Monolayers presenting trans-epithelial electric resistance (TEER) values below $300 \Omega \cdot \mathrm{cm}^{2}$ were excluded from the experiments. The 
resistance of the transport medium (HBSS) alone was considered as background resistance and subtracted from each TEER value $^{[17]}$.

Prior to the experiments, the culture medium was replaced with warm $\operatorname{HBSS}\left(37^{\circ} \mathrm{C}\right)$. The cell monolayer was equilibrated at $37^{\circ} \mathrm{C}$ for $30 \mathrm{~min}$ before conducting the transport studies. HBSS was removed and the test solutions (nanocrystal or coarse drug crystal suspension containing $50 \mu \mathrm{g} / \mathrm{mL}$ SQV in $\mathrm{HBSS}^{[18]}$ ) were added to the AP or BL (donor) compartments. At predetermined time points (30, 60, 90, and $120 \mathrm{~min}$ ), aliquots $(200 \mu \mathrm{L})$ were withdrawn from the receiver chamber, and an equivalent volume of HBSS was added to maintain a constant volume.

The drug concentration in the samples was determined by high performance liquid chromatography (HPLC) with the same method described below for analyzing plasma samples, and all experiments were performed in triplicate. The apparent permeability coefficient $\left(P_{\text {app }}, \mathrm{cm} / \mathrm{s}\right)$ was calculated using the following equation ${ }^{[19]}$ :

$$
P_{\text {app }}=\mathrm{d} Q / \mathrm{d} t \cdot 1 / A C_{0}
$$

where $\mathrm{d} Q / \mathrm{d} t$ is the transport rate $(\mu \mathrm{g} / \mathrm{s}), C_{0}$ is the initial drug concentration on the apical side $(\mu \mathrm{g} / \mathrm{mL})$, and $A$ is the surface area of the membrane filter $\left(1.12 \mathrm{~cm}^{2}\right)$.

\section{Histological examination of nanocrystal absorption in the intestinal mucosa}

The fluorescent-labeled nanocrystal suspensions were administered to rats at a dose of $50 \mathrm{mg} / \mathrm{kg}$ of SQV by intragastric gavage. After $1 \mathrm{~h}$, the animals were anesthetized by intraperitoneal injection of $25 \%$ urethane $(1 \mathrm{~g} / \mathrm{kg})$, and a $3 \mathrm{~cm}$ midline incision was made in the abdomen; segments of the duodenum, jejunum, and ileum of the small intestine were then identified. The proximal jejunum was located by identifying the ligament of Trietz; the distal jejunum and the beginning of the proximal ileum were located by visualizing an increase in the density of gut-associated lymphatic tissue (GALT); and the distal ileum was identified by locating the cecum. The identified segments were removed and washed extensively with a large amount of cold saline.

The isolated tissues were carefully everted, then embedded in TissueTek ${ }^{\circledR}$ O.C.T ${ }^{\text {TM }}$ Compound (Sakura Finetek, USA) at $-20^{\circ} \mathrm{C}$ and sectioned at $20 \mu \mathrm{m}$ (Leica CM1950, Germany). The sections were fixed with $4 \%$ buffered paraformaldehyde, stained with 4',6-diamidino-2-phenylindole (5 g/mL, Sigma) and imaged using CLSM (FluoView FV10i, Olympus, Japan).

\section{In vivo absorption study in rats Dosing}

Two groups of rats (4 per group) were orally administered two formulations, namely the coarse crystalline SQV suspension and nanocrystal SQV suspension, at a dose of $50 \mathrm{mg} / \mathrm{kg}$. The rats were allowed access to food at $4 \mathrm{~h}$ after the oral drug administration, and had free access to water during the entire experiment. Blood samples of $400 \mu \mathrm{L}$ were collected via the orbital venous plexus under isoflurane anesthesia at $0.5,1,1.5$, $2,3,4,6,8,10$, and $12 \mathrm{~h}$ after dosing. The whole blood was transferred into heparinized tubes, and the plasma was separated by centrifugation at $6500 \times g$ for $5 \mathrm{~min}$ and frozen at $-20^{\circ} \mathrm{C}$ until analysis.

\section{Plasma sample analysis}

The SQV concentrations in plasma were determined by HPLC. The samples for analysis were prepared by liquidliquid extraction with a slight modification ${ }^{[12]}$. Briefly, $30 \mu \mathrm{L}$ of an internal standard solution (ketoconazole $8 \mu \mathrm{g} / \mathrm{mL}$, in acetonitrile) and $150 \mu \mathrm{L}$ of $0.5 \mathrm{~mol} / \mathrm{L}$ sodium hydroxide were added to $150 \mu \mathrm{L}$ of rat plasma. The mixture was vortexed for $20 \mathrm{~s}$, and $0.5 \mathrm{~mL}$ of a methyl tert-butyl ether:dichloromethane mixture $(90: 10, v / v)$ was added to extract the drug. The extraction process was conducted three times. The organic layer was separated by centrifugation at $6500 \times \mathrm{g}$ for $5 \mathrm{~min}$ and transferred to another tube. Following solvent evaporation under a stream of nitrogen at $40^{\circ} \mathrm{C}$, the residue was reconstituted in $50 \mu \mathrm{L}$ of the mobile phase. The drug concentrations were analyzed with an Agilent 1100 series HPLC (Agilent Technologies, Santa Clara, CA, USA) with an Agilent Extend C18 column (3.5 cm, $250 \mathrm{~mm} \times 5 \mathrm{~mm}$; Agilent Technologies). The mobile phase contained acetonitrile and water $(55 / 45, v / v, \mathrm{pH} 7.5)$ with a flow rate of $1 \mathrm{~mL} / \mathrm{min}$. UV-detection was performed at $239 \mathrm{~nm}$. SQV quantification was based on the linear regression between the HPLC peak area ratio of SQV to the internal standard and the SQV concentration in plasma. Linearity was observed in the concentration range of $0.04-1.6 \mu \mathrm{g} / \mathrm{mL}$ with a correlation coefficient of 0.9946 . The limit of quantification (LOQ) for SQV in rat plasma was $40.0 \mathrm{ng} / \mathrm{mL}$. Intra-day and inter-day variability was below $10 \%$, and the recovery of SQV met the requirement for plasma sample analysis.

\section{Pharmacokinetic analysis}

Pharmacokinetic parameters were derived from drug concentration-time data with nonlinear modeling software (Phoenix ${ }^{\circledR}$ WinNonlin 6.3, Pharsight Corporation, USA) using a noncompartmental analysis model. The parameters determined include the maximal plasma concentration of the drug $\left(C_{\max }\right)$, the time taken to reach the maximum plasma concentration $\left(T_{\max }\right)$, and the area under the plasma drug concentration-time curve $\left(\mathrm{AUC}_{0-12 \mathrm{~h}}\right)$.

\section{Statistical analysis}

The experimental data were analyzed using Microsoft Excel 2013 (Microsoft, Redmond, WA, USA). Student's $t$-test was used to determine the significance of differences between the two groups. The difference was considered to be statistically significant if the probability $(P)$ value was less than 0.05 $(P<0.05)$ and to be very significant if the $P$ value was less than $0.01(P<0.01)$. All data were expressed as the mean \pm standard deviation (SD) unless otherwise indicated.

\section{Results}

\section{Nanocrystal characterization}

Colloidal SQV nanocrystals were prepared by an anti-solvent precipitation-high pressure homogenization method ${ }^{[20]}$. The 
nanocrystals had a particle size of $205.93 \pm 3.74 \mathrm{~nm}$ with a polydispersity index (PDI) of approximately 0.1, indicating a uniform distribution as shown in Figure 1A. The zeta potential of the SQV nanocrystals was $-53.5 \pm 2.42 \mathrm{mV}$ (Figure 1B). The zeta potential is an important characteristic of the electrical double layer and is a key parameter that is widely used to predict the stability of suspensions. In general, the higher the absolute value of the zeta potential, the more stable the suspension ${ }^{[4]}$. The high zeta potential of the SQV nanocrystals indicates the high degree of stability of the formulation.

In the pilot study, nanocrystal flocculation was observed when non-ionic stabilizers, such as hydroxypropyl methylcellulose, D-alpha tocopheryl polyethylene glycol 1000 succinate, and polyvinylpyrrolidone, were used. In this study, we used poly(sodium 4-styrenesulfonate) (PSS), an anionic polymer, as the stabilizer. PSS was adsorbed onto the surface of the nanocrystals, thereby providing steric hindrance and electrostatic repulsion between the particles ${ }^{[4]}$. A pilot study indicated that $0.12 \%$ PSS was sufficient to stabilize the nanocrystals in suspension for several months at $4^{\circ} \mathrm{C}$ (data not shown).

The particle morphology of the coarse powder was examined under an SEM. The coarse powder presented an irregular shape and a particle size of approximately 2-5 $\mu \mathrm{m}$, as shown in Figure 2A. By contrast, the examination of the nanocrystals by TEM revealed rod-shaped particles, as shown in Figure 2B. The length of the nanocrystals measured by TEM was in agreement with the particle size measured by the Zetasizer.

The XRPD was used to determine the crystallinity of the SQV nanocrystals. As shown in Figure 3, the crystalline form of the SQV nanocrystals was identical to that of the coarse powder. However, the lower intensity observed for the nanocrystals may be attributed to the lower crystallinity of the samples.

\section{Aqueous solubility and dissolution of the nanocrystals}

The solubility of the nanocrystals in PBS ( $\mathrm{pH} 6.8$ ) was $40.85 \pm 11.36 \mu \mathrm{g} / \mathrm{mL}$, which was lower than the solubility of the coarse powder $72.61 \pm 17.86 \mu \mathrm{g} / \mathrm{mL}$. This difference in solubility was most likely because, while the coarse drug powder was in the mesylate salt form, the drug could have been converted to the free base during the preparation of the nanocrystal formulation. The dissolution profiles of the nanocrystals and the coarse crystals are shown in Figure 4 . Only $20 \%$ of the coarse crystals were dissolved during a $2 \mathrm{~h}$ test period, while approximately $60 \%$ dissolution was observed for the SQV nanocrystals. A state of equilibrium was achieved in $30 \mathrm{~min}$. The results demonstrate that the dissolution rate of the SQV nanocrystals is faster than the coarse powder. The promising results of the drug release studies prompted cellular and in vivo studies in rats with the nanocrystal formulation.

The SQV crystals were stained by fluorescein ethyl rhodamine B. The release profiles of ethyl rhodamine B from the SQV nanocrystals and the coarse crystals were also determined. As shown in Figure 4, the release percentage of ethyl rhodamine B from the coarse SQV crystals was lower than the SQV nanocrystals, and the release of ethyl rhodamine B from
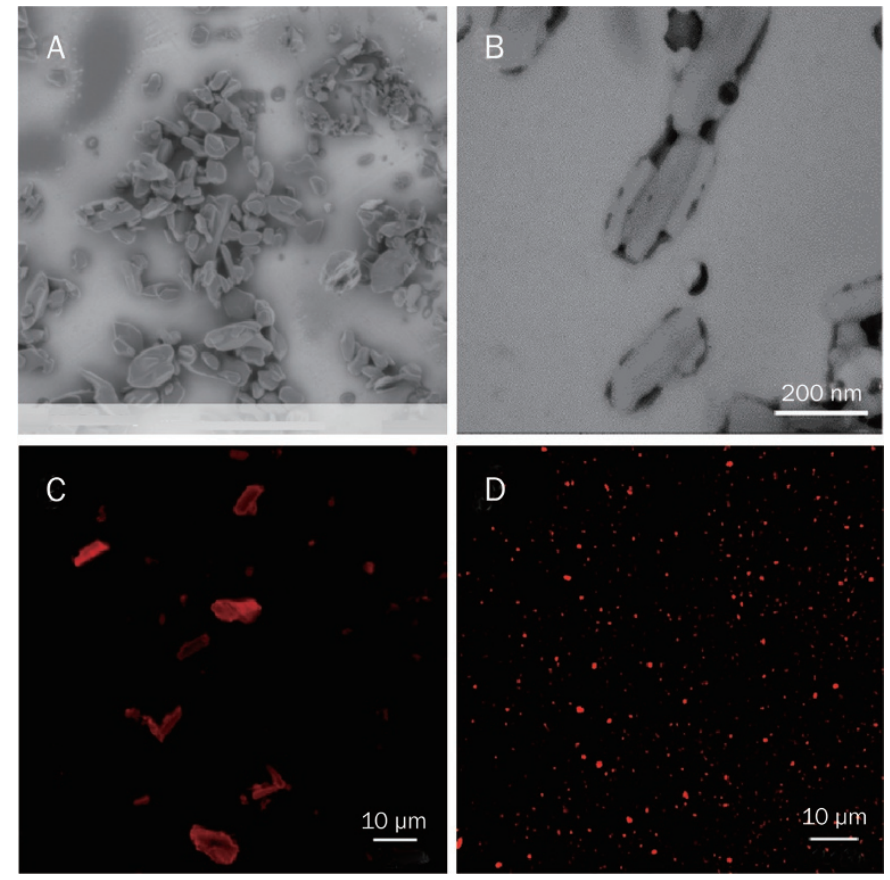

Figure 2. Morphology of coarse saquinavir (SQV) crystals (A) and SQV nanocrystals (B) observed under a scanning electron microscope and transmission electron microscope, respectively; Fluorescent-labeled coarse SQV crystals (C) and nanocrystals (D), as observed under a confocal laser scanning microscope.

the two types of crystal showed the same trend as that of SQV from the corresponding crystals. The results demonstrated that ethyl rhodamine B was entrapped in the SQV crystal lattice and released from the crystals as the SQV crystals dissolved.

Figure 2C and 2D show the CLSM images of the ethyl rhodamine B stained coarse crystals and nanocrystals. Individual SQV coarse crystals and nanocrystals were clearly observed under high magnification (60× objective lens), demonstrating that the fluorescent intensity of an individual crystal was significantly higher than the free fluorescent molecules, although some fluorescent molecules may be dissolved in the medium. Cellular uptake and the intestinal mucosa absorption of the SQV crystals were subsequently studied using the ethyl rho-

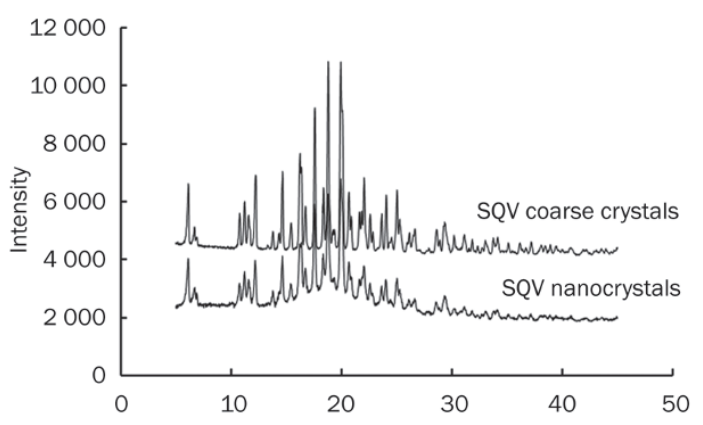

Figure 3. X-ray powder diffraction (XRPD) characterization of saquinavir (SQV) coarse crystals and nanocrystals. 


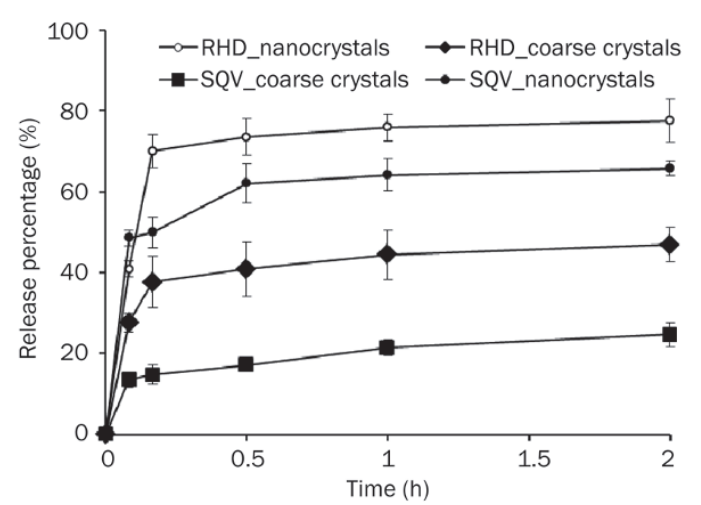

Figure 4. Dissolution profiles of saquinavir (SQV) and ethyl rhodamine $B$ (RHD) from nanocrystals and coarse crystals $(n=3)$.

damine B stained SQV crystals.

Cellular uptake of SQV in Caco-2 cells and its transport across Caco-2 cell monolayers

Figure 5A shows the CLSM images of Caco-2 cells incubated with the SQV nanocrystal suspension and the coarse SQV crystal suspension for different lengths of time. The fluorescent intensity of the SQV nanocrystal suspension was higher than the coarse crystals, indicating increased drug uptake. After $2 \mathrm{~h}$, individual high-intensity red fluorescent particles could be observed in the cytoplasm of the cells exposed to the nanocrystal suspension. These particles were most likely to be "the nanocrystals." However, very few individual particles were observed in the cytoplasm of the cells exposed to the coarse crystal suspension detected at the same excitation intensity and exposure time as that of nanocrystals. The fluorescence intensity of the cells incubated with nanocrystals was 2.19-fold higher than the coarse crystals, and revealed the significant advantage of nanocrystal uptake by the Caco-2 cells (Figure 5B).

To evaluate the effect of the particle size of the crystals (coarse crystals $v s$ nanocrystals) on SQV transport through cell monolayers, we determined the drug transport across Caco-2 cell monolayers from the AP side to the BL side. In all the experiments, the TEER measurements showed no cellular damage. Figure 6A shows the time profiles of SQV permeation through the Caco- 2 cell monolayers. The amount of drug in the receiving chamber (BL side) increased with time for both formulations. It was clear that the amount of drug in the receiving chambers of monolayers treated with the nanocrystals was much higher than the amount in the receiving chambers of monolayers treated with the coarse crystals at each time point. These results indicated that the drug transport for the nanocrystals was faster than the coarse crystals.

The $P_{\text {app }}$ of SQV from the apical side to the basolateral side was calculated after incubating Caco- 2 cells with the two formulations for 0.5 to $2.0 \mathrm{~h}$. As shown in Figure 6B, the $P_{\text {app }}$ of the nanocrystal SQV formulation $\left[(4.878 \pm 0.434) \times 10^{-6} \mathrm{~cm} / \mathrm{s}\right]$ was significantly higher than the corresponding value of the coarse SQV crystals $\left[(2.250 \pm 0.437) \times 10^{-6} \mathrm{~cm} / \mathrm{s} ; P=0.0018\right]$.

\section{Bioimaging study of SQV nanocrystal absorption in the intestine}

To study the uptake of SQV nanocrystals in intestinal tract, the nanocrystals were labeled with ethyl rhodamine $B$, and the intestinal mucosal absorption was studied by CLSM. Figure 7 shows the representative CLSM images of different intestinal segments (duodenum, jejunum, and ileum) at $1 \mathrm{~h}$ after oral administration of fluorescein labeled SQV nanocrystals and coarse SQV crystals. The crystals in the intestinal lumen were
A
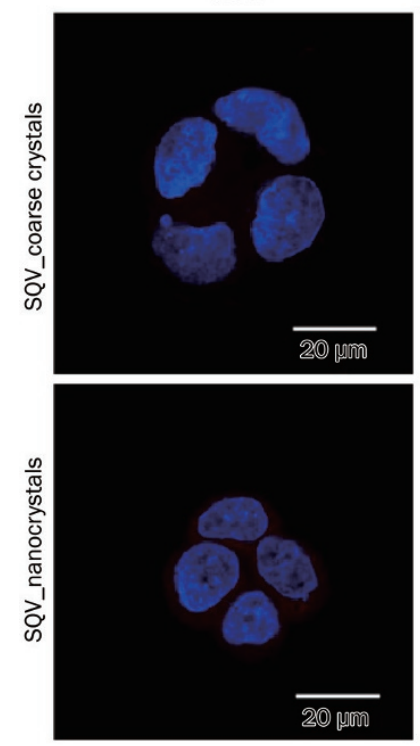

$1 \mathrm{~h}$
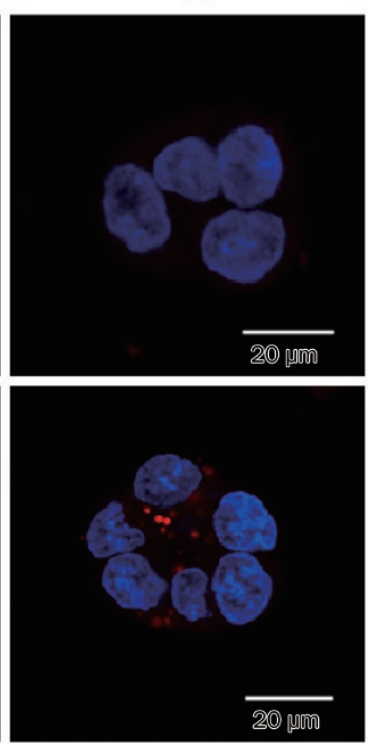

$2 \mathrm{~h}$

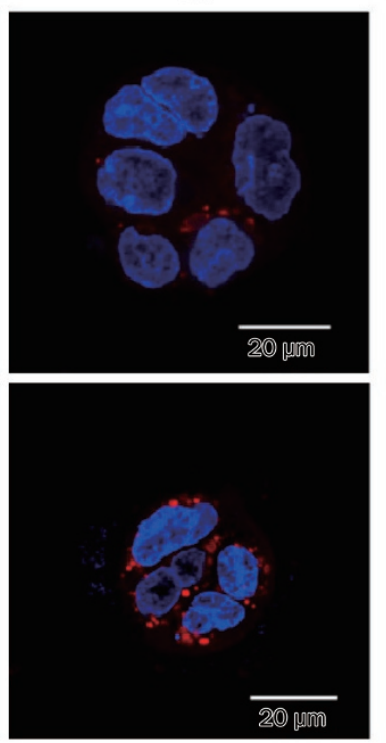

B

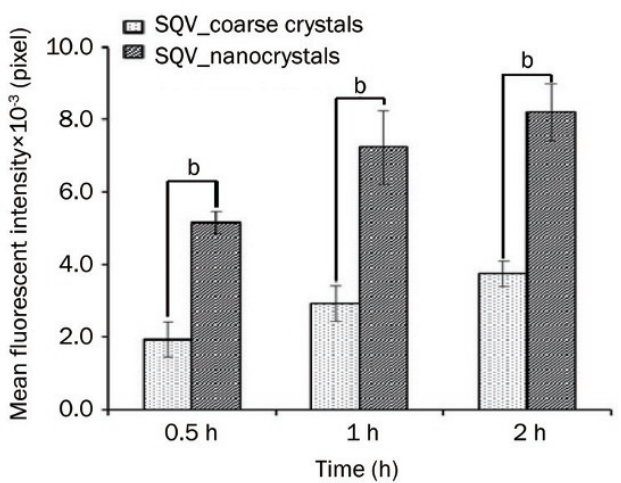

Figure 5. (A) Cellular uptake of saquinavir (SQV) coarse crystals and nanocrystals at different time points observed under confocal laser scanning microscope; (B) quantitatively analysis of fluorescence intensity of cells $(n=3)\left({ }^{b} P<0.05\right)$. 
removed by extensive washing with large amounts of cold saline. The red fluorescent signals observed indicate the nanocrystals or diffuse fluorescein uptake by the intestinal epithelia cells.

As shown in Figure 7, the coarse SQV crystals were not completely dissolved in the gastrointestinal tract $1 \mathrm{~h}$ after administration. Some large coarse SQV crystals were clearly observed on the luminal side of the intestinal tract (indicated by white arrows in the coarse SQV images). This is presumably because the sink condition is difficult to attain due to the low solubility and absorption rate for the coarse SQV crystals. The fluorescent intensity was extremely low in the epithelial cells, indicating that the drug uptake from the coarse SQV crystals was inefficient. For the nanocrystal SQV formulation, the images showed that strong fluorescent signals were observed in the epithelial cells (EC) and the lamina propria (LP) of the duodenum and jejunum, as indicated by the yellow arrows in the nanocrystal group in Figure 7. The intensity of the fluorescent signals was higher than those observed for the coarse SQV crystals. These results demonstrate that the nanocrystals entered into the epithelial cells more easily than the coarse crystals. The low fluorescent intensity of the ileum (lower part of intestinal tract) for both suspensions may be due to the slow gastric emptying rate in rats and an insufficient time for intestinal transit ${ }^{[21]}$.

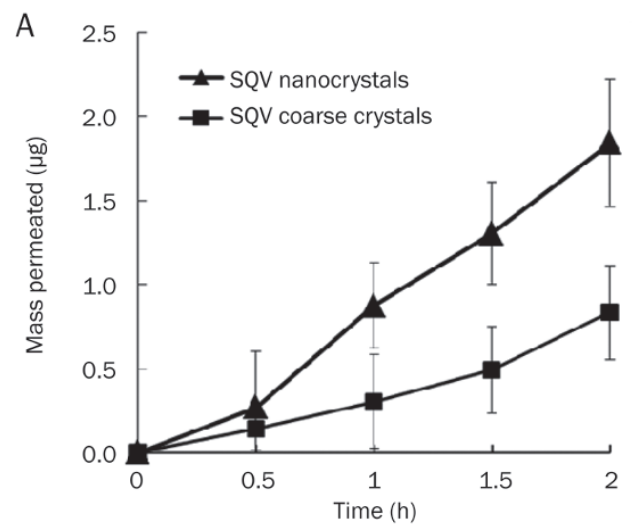

B

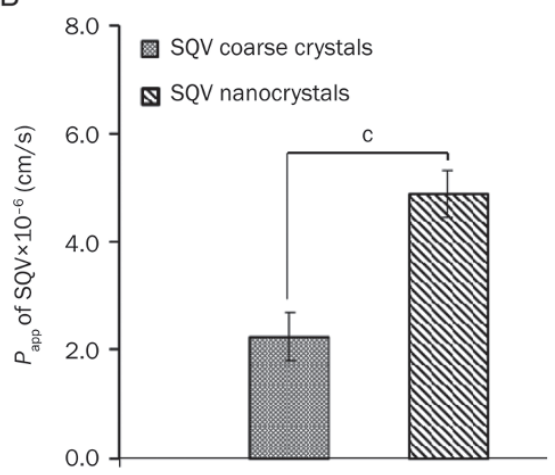

Figure 6. (A) Time-profiles of permeation of saquinavir (SQV) through a monolayer of Caco-2 cells; (B) Apparent permeability coefficient $\left(P_{\text {app }}\right)$ of SQV across a Caco- 2 cell monolayer $(n=3) .{ }^{C} P<0.01$.

\section{Pharmacokinetic studies of SQV in rats}

The pharmacokinetics of SQV were studied in rats to assess the absorption efficiency of the nanocrystals. The pharmacokinetic parameters are shown in Table 1, and the plasma drug concentration-time profiles of SQV are presented in Figure 8. Following oral administration, there were significant differences in the pharmacokinetic profiles of the SQV nanocrystals and coarse SQV crystals. The $C_{\max }$ of the nanocrystals was 2.16 -fold of that for coarse crystals $(P<0.01)$. The AUC for the SQV nanocrystals was 1.95-fold of that for the $\mathrm{AUC}_{(0-12 \mathrm{~h})}$ for coarse crystals $(P<0.01)$.

\section{Discussion}

Saquinavir is a very important class of anti-HIV drugs, however the poor solubility and low bioavailability of this drug has presented a substantial challenge to pharmaceutical scientists. During our research, we prepared a nanocrystal SQV formulation by an anti-solvent precipitation-high pressure homogenization method. This method has two important steps include precipitation and homogenization. If the precipitated nanoparticle suspension was not subjected to highpressure homogenization, the nanoparticles were found to be extremely unstable and subsequently grew into micro-particles within a day. It is likely that the amorphous SQV nanoparticles were obtained by anti-solvent precipitation at $4{ }^{\circ} \mathrm{C}$. Even with the addition of a stabilizer, the amorphous nanoparticle suspension was extremely unstable under ambient conditions. The amorphous to crystalline transformation progressed with an increase in particle size and a change in polymorphism, causing the precipitated drug particles to develop into large particles within a few days ${ }^{[22-24]}$. High-pressure homogenization has been shown to convert materials from thermodynamically unstable amorphous particles into more stable crystalline nanoparticles ${ }^{[3,25]}$. As a result, SQV nanocrystals with a particle size of approximately $200 \mathrm{~nm}$ and a uniform particle size distribution (PDI $\sim 0.1$ ) were successfully prepared with the combination of anti-solvent precipitation and high-pressure homogenization.

Though the dissolution studies, we find that the dissolution rate of nanocrystals was faster that the coarse powder. The enhanced dissolution rate can be attributed to the increased surface area of nanocrystals available for interaction with the solvent and the decreased diffusion layer thickness of the nanocrystals compared with the coarse crystals ${ }^{[3]}$. As submicron particles can easily pass through a line filter, overestimation of the dissolution rate is possible. However, visual observation of the immediate disappearance of turbidity in the dissolution vessel suggests that the solid particles that passed through the filter were of little consequence. Although the dissolution rate of SQV nanocrystals was faster than the coarse powder, only approximately $60 \%$ dissolution was observed for the nanocrystals. There is a high probability that the dissolution medium was completely saturated by the dissolved drug because the solubility of the nanocrystals is lower than the coarse powder.

It is reported that particles with a size of $200 \mathrm{~nm}$ or smaller 

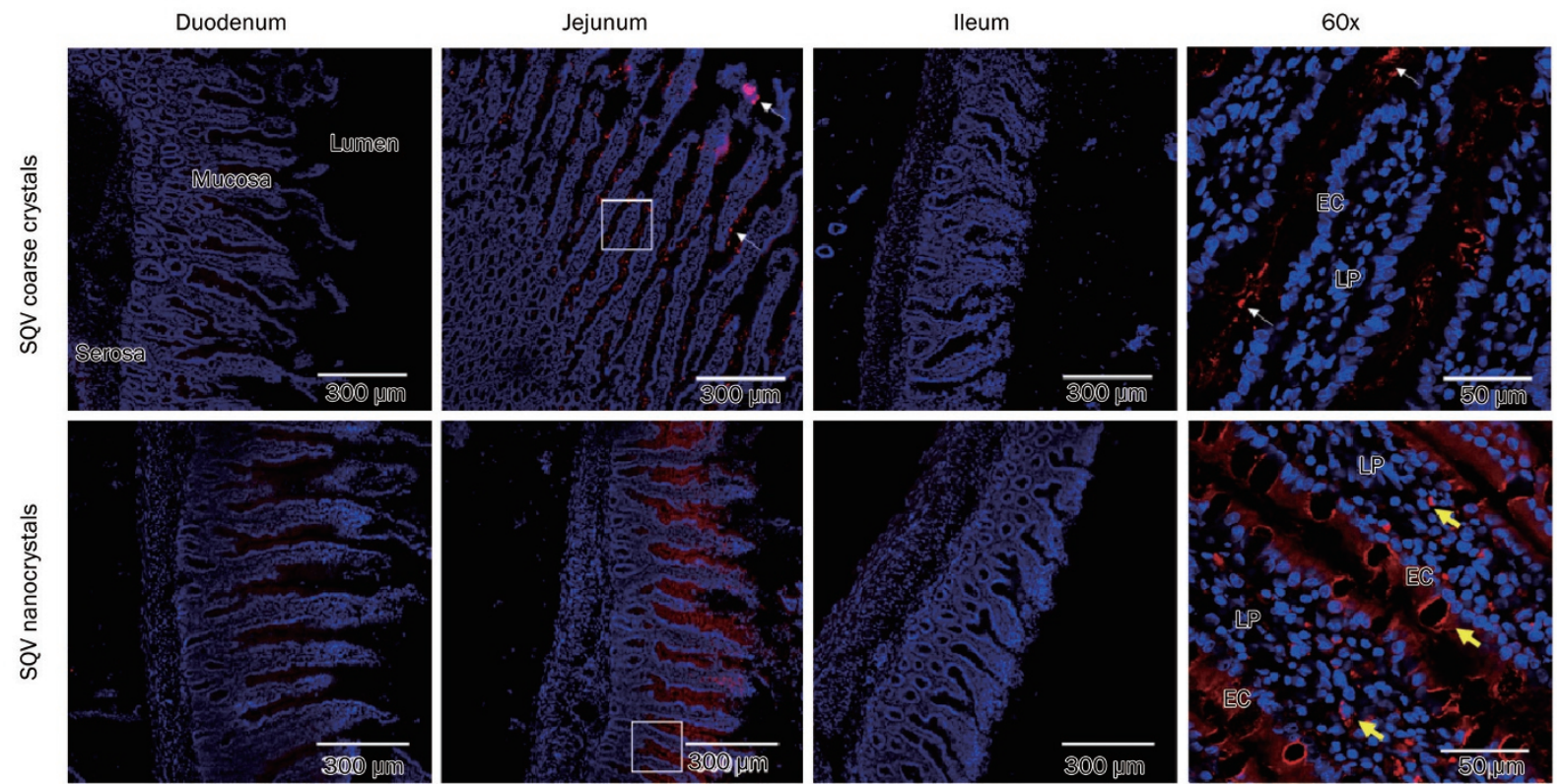

Figure 7. Histological examination of the absorption of saquinavir (SQV) nanocrystals and coarse crystals in the intestinal mucosa $1 \mathrm{~h}$ after oral administration. The figures in the right column are the magnified images of white rectangular regions on the left. Lamina propria (LP), intestinal epithelium cells (EC).

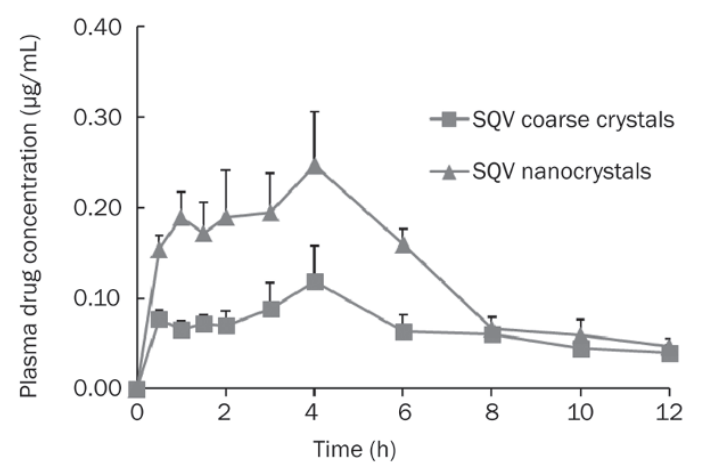

Figure 8. Plasma drug concentration-time profiles of saquinavir (SQV) after oral administration of SQV coarse crystals and nanocrystals $(n=4)$.

may be preferentially internalized via the clathrin-coated pits $^{[26]}$. Therefore, in addition to passive diffusion of the dissolved free molecules across the cell membrane, the SQV nanocrystals may be directly taken up by the cells via multiple endocytosis pathways, which may include clathrin-mediated or caveolae-mediated pathways ${ }^{[27]}$. The enhanced transport of the nanocrystal SQV formulation across the Caco-2 monolayer was likely due to the increased cellular uptake of the nanocrystals, as shown in previous experiments in this study. The efficient transport of SQV across Caco-2 monolayer was increased by the enhanced release of SQV from the nanocrystals, and the rapid accumulation of the drug in cells was caused by the potential uptake of individual nanocrystals via multiple endocytosis pathways ${ }^{[27]}$.

When pharmacokinetic studies were conducted in male Wistar rats, a significant increase of SQV plasma concentration was observed when the drug was administered orally in nanocrystals. The enhanced oral absorption of the SQV nanocrystals was a result of the increased drug dissolution rate and improved epithelial uptake via multiple potential endocytosis pathways, including clathrin-mediated or caveolae-mediated pathways. It has been reported that $\mathrm{SQV}$ is the substrate for P-gp, which may also greatly limit its intestinal uptake. In addition, the hepatic and intestinal first pass metabolism may also contribute to its low bioavailability ${ }^{[11,14]}$. The nanocrystal formulation technique, in combination with P-gp inhibition and avoidance of intestinal and hepatic first pass effects, may

Table 1. Pharmacokinetic parameters of SQV after oral administration of coarse crystals and nanocrystals.

\begin{tabular}{lcccc}
\hline Formulations & $T_{\max }$ & $C_{\max }$ & $\mathrm{AUC}_{(0-12)}$ & $\mathrm{AUC}_{(0-\infty)}$ \\
\hline Coarse crystals & $3.750 \pm 0.433$ & $0.123 \pm 0.037$ & $0.798 \pm 0.177$ & $1.242 \pm 0.126$ \\
Nanocrystals & $3.375 \pm 1.083$ & $0.266 \pm 0.028$ & $1.556 \pm 0.154^{\circ}$ & $1.757 \pm 0.178$ \\
\hline
\end{tabular}

AUC, the area under the blood drug concentration-time curve; $C_{\max }$, the maximal blood concentration of drug; $T_{\max }$, the time taken to reach the $C_{\max }$. ${ }^{c} P<0.01$ for the comparison between coarse crystals and nanocrystals, Student's $t$-test. 
further improve the bioavailability of SQV. These factors are a major focus of our future studies.

\section{Conclusion}

In this study, SQV nanocrystals were formulated to improve oral drug absorption. The particle size, dissolution, cellular uptake, and transport across Caco-2 cell monolayers were studied. The uptake of SQV nanocrystals in the intestinal tract was imaged ex vivo, and oral drug absorption was examined in vivo. The results showed that oral absorption of SQV was significantly improved by increased cellular uptake and transport resulting from enhanced drug dissolution and the cellular uptake of individual nanocrystals compared with the coarse crystals. However, to further improve the bioavailability of $S Q V$, the nanocrystal formulation technique in combination with P-gp inhibition and avoidance of intestinal and hepatic first pass effects are needed, and these factors are a major focus of our future studies.

\section{Acknowledgements}

This work was supported by the National Natural Science Foundations of China (grant No 81202468 and 81373356) and the China Postdoctoral Science Foundations (2013M530219 and 2014T70445). This work was also partially supported by the Shanghai Science and Technology Innovation Action Plan for Basic Research (14JC1493200). Deng-ning XIA also gratefully acknowledges the support of the SA-SIBS scholarship program.

\section{Author contribution}

Yuan HE and Deng-ning XIA had full access to all the data in the study and take responsibility for the integrity of the data and the accuracy of the data analysis; Deng-ning XIA conceived and designed the study; Yuan HE, Qiu-xia LI, and Jinsong TAO performed the experiments; Yuan HE and Dengning XIA drafted the manuscript; Chi WANG and Yong GAN revised the manuscript; and Deng-ning XIA and Yong GAN obtained funding.

\section{References}

1 Babu NJ, Nangia A. Solubility advantage of amorphous drugs and pharmaceutical cocrystals. Cryst Growth Des 2011; 11: 2662-79.

2 Kesisoglou F, Panmai S, Wu Y. Nanosizing-oral formulation development and biopharmaceutical evaluation. Adv Drug Delivery Rev 2007; 59: 631-44.

3 Xia D, Quan P, Piao H, Piao H, Sun S, Yin Y, et al. Preparation of stable nitrendipine nanosuspensions using the precipitation-ultrasonication method for enhancement of dissolution and oral bioavailability. Eur J Pharm Sci 2010; 40: 325-34.

4 Xia D, Gan Y, Cui F. Application of precipitation methods for the production of water-insoluble drug nanocrystals: production techniques and stability of nanocrystals. Curr Pharm Des 2014; 20: 408-35.

5 Cartiera MS, Johnson KM, Rajendran V, Caplan MJ, Saltzman WM. The uptake and intracellular fate of PLGA nanoparticles in epithelial cells. Biomaterials 2009; 30: 2790-8.

6 Hu L, Huang B, Zuo MM, Guo RY, Wei H. Preparation of the phycoerythrin subunit liposome in a photodynamic experiment on liver cancer cells. Acta Pharmacol Sin 2008; 29: 1539-46.

7 Kuo YC, Wang CC. Cationic solid lipid nanoparticles with primary and quaternary amines for release of saquinavir and biocompatibility with endothelia. Colloids Surf B Biointerfaces 2013; 101: 101-5.

8 Xia D, Cui F, Piao H, Cun D, Piao H, Jiang Y, et al. Effect of crystal size on the in vitro dissolution and oral absorption of nitrendipine in rats. Pharm Res 2010; 27: 1965-76.

9 Fu Q, Sun J, Ai X, Zhang P, Li M, Wang Y, et al. Nimodipine nanocrystals for oral bioavailability improvement: role of mesenteric lymph transport in the oral absorption. Int J Pharm 2013; 448: 290-7.

10 Griffin BT, O'Driscoll CM. An examination of the effect of intestinal first pass extraction on intestinal lymphatic transport of saquinavir in the rat. Pharm Res 2008; 25: 1125-33.

11 Perry CM, Noble S. Saquinavir soft-gel capsule formulation - a review of its use in patients with HIV infection. Drugs 1998; 55: 461-86.

12 Pathak SM, Musmade P, Dengle S, Karthik A, Bhat K, Udupa N. Enhanced oral absorption of saquinavir with Methyl-Beta-CyclodextrinPreparation and in vitro and in vivo evaluation. Eur J Pharm Sci 2010; 41: 440-51.

13 Branham ML, Moyo T, Govender T. Preparation and solid-state characterization of ball milled saquinavir mesylate for solubility enhancement. Eur J Pharm Biopharm 2012; 80: 194-202.

14 Aungst BJ. P-glycoprotein, secretory transport, and other barriers to the oral delivery of anti-HIV drugs. Adv Drug Deliv Rev 1999; 39: 105-16.

15 Zhao R, Hollis CP, Zhang H, Sun L, Gemeinhart RA, Li T. Hybrid nanocrystals: achieving concurrent therapeutic and bioimaging functionalities toward solid tumors. Mol Pharm 2011; 8: 1985-91.

16 Beriault DR, Werstuck GH. Detection and quantification of endoplasmic reticulum stress in living cells using the fluorescent compound, Thioflavin T. Biochim Biophys Acta 2013; 1833: 2293-301.

$17 \mathrm{Li} \mathrm{YH,} \mathrm{Bi} \mathrm{HC,} \mathrm{Huang} \mathrm{L,} \mathrm{Jin} \mathrm{J,} \mathrm{Zhong} \mathrm{GP,} \mathrm{Zhou} \mathrm{XN,} \mathrm{et} \mathrm{al.} \mathrm{Phorbol}$ 12-myristate 13-acetate inhibits P-glycoprotein-mediated efflux of digoxin in MDCKII-MDR1 and Caco-2 cell monolayer models. Acta Pharmacol Sin 2014; 35: 283-91.

18 Beloqui A, Solinis MA, Gascon AR, Del Pozo-Rodriguez A, des Rieux A, Preat V. Mechanism of transport of saquinavir-loaded nanostructured lipid carriers across the intestinal barrier. J Control Release 2013; 166: 115-23.

19 Waterbeemd Hvd, Lennernäs H, Artursson P, editors. Drug bioavailability: estimation of solubility, permeability, absorption, and bioavailability. WILEY-VCH; 2003.

20 Quan P, Shi K, Piao HZ, Piao HY, Liang N, Xia DN, et al. A novel surface modified nitrendipine nanocrystals with enhancement of bioavailability and stability. Int J Pharm 2012; 430: 366-71.

21 Roger E, Lagarce F, Garcion E, Benoit JP. Biopharmaceutical parameters to consider in order to alter the fate of nanocarriers after oral delivery. Nanomedicine (Lond) 2010; 5: 287-306.

22 Xia D, Wu JX, Cui F, Qu H, Rades T, Rantanen J, et al. Solventmediated amorphous-to-crystalline transformation of nitrendipine in amorphous particle suspensions containing polymers. Eur J Pharm Sci 2012; 46: 446-54.

23 Matteucci ME, Paguio JC, Miller MA, Williams lii RO, Johnston KP. Flocculated amorphous nanoparticles for highly supersaturated solutions. Pharm Res 2008; 25: 2477-87.

24 Matteucci ME, Brettmann BK, Rogers TL, Elder EJ, Williams RO 3rd, Johnston KP. Design of potent amorphous drug nanoparticles for rapid generation of highly supersaturated media. Mol Pharm 2007; 4: 782-93.

25 Quan P, Xia DN, Piao HZ, Piao HY, Shi K, Jia YN, et al. Nitrendipine nanocrystals: its preparation, characterization, and in vitro-in vivo 
evaluation. AAPS PharmSciTech 2011; 12: 1136-43.

26 Rejman J, Oberle V, Zuhorn IS, Hoekstra D. Size-dependent internalization of particles via the pathways of clathrin- and caveolae- mediated endocytosis. Biochem J 2004; 377: 159-69.

27 Zhang H, Hollis CP, Zhang Q, Li T. Preparation and antitumor study of camptothecin nanocrystals. Int J Pharm 2011; 415: 293-300. 